\title{
CATEGORY OF FUZZY SETS
}

\author{
Bidyanand Prasad
}

Department of Mathematics

\begin{abstract}
In the present paper, the concept of category theory, the category of fuzzy sets and the theory of flou sets are introduced. This article also shows the isomorphism between the category of fuzzy sets and flou sets.
\end{abstract}

\section{Keywords}

Category, flou sets, fuzzy sets, morphism, functor

\section{Introduction}

The application of algebra in geometry brings out certain strong analogies between the two subjects. The continuous mapping form one space into another corresponds to homomorphism of the associated groups. If the topological space is a surface, it may have a tangent plane, which has the structure of a vector space. Then a mapping from one surface to another which is a linear transformation of the corresponding tangent spaces. Such anologies led to a new subject of study known as category theory. Category theory is developed in 1942 in the work of Sounders MacLane(1909-1995) and Samuel Eileanberg(b.1913).

\section{Category theory}

In category theory the basic elements are sets of 'objects' and mapping among the objects called 'morphism' . The objects in category theory may be vector spaces and morphim may be linear transformation. Also the objects may be topological spaces and the morphism may be continuous function. All these particular objects are in compassed in the more general subjects of category theory (Cooke, 1997).

A category $K$ is a domain of mathematical discourse comprising a collection of objects, for each pair (A, B) of objects a collection $K$ (A, B) of morphisms:

$f: A \rightarrow B$ or $A \stackrel{f}{\rightarrow} B$ with domain $\mathrm{A}$ and codomain $\mathrm{B}$, together with a law of composition:

$$
g . f: A \rightarrow C
$$

where $f: A \rightarrow B \& g: B \rightarrow C$, which is associative and has identities $\mathrm{id}_{\mathrm{A}}: \mathrm{A} \rightarrow \mathrm{A}$ 


\section{B. Prasad}

A functor $\mathrm{H}$ from category $K$ to category $L$ sends objects A to objects $\mathrm{AH}$ and morphisms $f: A \rightarrow B$ in $K$ to morphisms $f H: A H \rightarrow B H$ in $L$ as

If $f=i d_{A}: A \rightarrow A$ then

$f H=i d_{A H}: A H \rightarrow A H g$ and

if $f=\mathrm{A} \stackrel{g}{\rightarrow} \mathrm{B} \stackrel{h}{\rightarrow} \mathrm{C}$ then

$f H=A H \stackrel{g H}{\rightarrow} B H \stackrel{h H}{\rightarrow} C H$.

\section{Category of fuzzy sets}

The theory fuzzy sets extended the basic mathematical concepts of set. In view of the fact that set theory is the corner-stone of modern Mathematics a new and more general framework of mathematics was established. Fuzzy mathematics is just a kind of mathematics developed in theis framework and a fuzzy topology is just a kind of topology developed on fuzzy sets. Hence, fuzzy mathematics being such kind of mathematical theory which contains wider content than the classical theory inspired the author to work on fuzzy topological spaces (Zadeh, 1965).

Let SET (l) be the category of fuzzy sets whose objects are pairs $(\mathrm{X}, \mathrm{A})$

where $\mathrm{X}$ is a set and $A \in I^{X}$.

A morphism $(X, A) \stackrel{\Phi}{\rightarrow}(Y, B)$ is a function

$\Phi: X \rightarrow Y$ such that ВoФ : $X \rightarrow I$ with ВОФА.

It is also possible to represent SET (1) with the category of Flou sets defined by :

\section{Definition}

A flou (vegue) set of a set $\mathrm{X}$ is a mapping $f: I \rightarrow P(X)$ provided

(i) $f$ is non increasing with $f(0)=1 \& f(1)=0$.

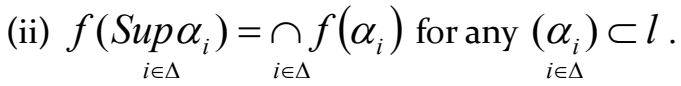

\section{Remarks}

For a Flou set, $A \subset f_{f}(\lambda) \Rightarrow \exists \lambda^{*}>\lambda$
such that $A \subset f\left(\lambda^{*}\right)$

There is a complete isomorphism between $I^{X} \& P(X)^{I}$.

\section{Theorem}

Modelling theorem : A mapping $t: P(X)^{I} \rightarrow I^{X}$ is bijective s.t.

$f(\lambda)$ is the $\lambda$ th cut of $\mathrm{tf}$ provided

$t f=\left\{\begin{array}{l}\vee\{\lambda \mid x \in f(\lambda)\} \text { for } x \in f(0) \\ 0, \quad \text { otherwise. }\end{array}\right.$

Proof : I. $\quad X \in(f)_{\lambda} \Rightarrow f(X)>\lambda$

$$
\Rightarrow \exists \lambda^{*}>\lambda
$$

with $f(x)>\lambda^{*}$

$\Rightarrow x \in(f) \lambda^{*} \Rightarrow x \in f\left(\lambda^{*}\right) \subset f(\lambda) \Rightarrow(f)_{\lambda} \subset f(\lambda)$

$$
\text { Also, } \quad x \in f(\lambda) \Rightarrow \exists \lambda^{*}
$$

with $x \in f\left(\lambda^{*}\right) \Rightarrow f(x)>\lambda^{*}>\lambda$

$$
\begin{aligned}
& \Rightarrow X \in(f)_{\lambda} \\
& \Rightarrow f(\lambda) \subset(f)_{\lambda}
\end{aligned}
$$

$$
\text { II. } \quad \begin{aligned}
f_{1}=f_{2} & \Rightarrow\left(f_{1}\right)_{\lambda}=\left(f_{2}\right)_{\lambda} \\
& \Rightarrow f_{1}(\lambda)=f_{2}(\lambda) \\
& \Rightarrow f_{1}=f_{2}
\end{aligned}
$$

\section{Remarks}

For Lucasiewicz $W_{3}=\{0,1 / 2,1\}$, Flou set can be identified with a pair:

$(\mathrm{A}, \mathrm{B})$ where $\mathrm{A}, \mathrm{B}$ are crisp subsets of X with $A \subseteq B$. 


\section{Theorem}

The category SET (l) \& Fl (l) are isomorphic.

Proof : Let the objects of SET (l) be pairs (X, A) whose morphisms $(X, A) \stackrel{\Phi}{\rightarrow}(Y, B)$ are functions $\Phi: X \rightarrow Y$ s.t. $B 0 \Phi: X \rightarrow I$ where $B 0 \Phi \geq A$. Let the category of vegue sets be $\mathrm{Fl}(\mathrm{l})$, the objects of which are the pairs $(\mathrm{X}, \mathrm{f})(f \in p(X))$ whose morphisms $(X, f) \stackrel{\Psi}{\rightarrow}(Y, g)$ are functions $\Psi: X \rightarrow Y$, s.t $\Psi(f(\alpha)) \subseteq g(\alpha)$.

It is possible to build a pair of functors SET

$\ddot{\mathbf{O}}$

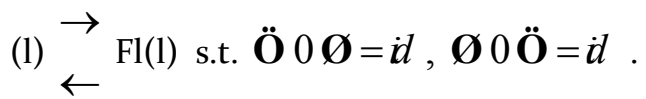

$\varnothing$

For SET (1), $\ddot{\mathbf{O}}(X, A)=\left(X, f_{A}\right)$, where

$$
f_{A}(\alpha)=\{\mathbf{x} \in \mathbf{X} \mid A(\mathbf{x}) \geq \alpha\} \forall \alpha \in \mathbf{I},
$$

and

$$
\boldsymbol{\varnothing}(X, f)=\left(X, A_{f}\right) \text {, where }
$$

$A_{f}(X)=\sup \{\alpha \in I \mid X \in f(a)\} \forall x \in X \quad$, and

$$
\ddot{\mathbf{O}}(\Phi)=\Phi, \boldsymbol{\varnothing}(\Psi)=\Psi \text {. }
$$

It can easily be verified, using modelling theorem that the pair of funtors $(\ddot{\mathbf{O}} \boldsymbol{\emptyset})$ leads to categorical isomorphism.

\section{Remarks}

If $L$ is a complete lattice and $\sum$ is a Heyting algebra then SET (1) can be generalized to SET (L) \& SET $\left(\sum\right)$.

\section{References}

Cooke, R. (1997). The history of mathematics: a brief course. New York: John Wiley and Sons, Inc.

Zadeh. L.A. (1965). Fuzzy sets. Information and control, 8, 338-353.

\section{The Author}

Bidyanand Prasad is Lecturer in the Department of Mathematics, Thakur Ram Multiple Campus, Birgunj. He obtained Ph. D. from Baba Ambedkar Bihar Univeristy, Muzaffarpur(India). He has been teaching different courses in Mathematics for the last seventeen years. His area of interest includes Differential Geometry and Complex Analysis. 